This item was submitted to Loughborough's Research Repository by the author.

Items in Figshare are protected by copyright, with all rights reserved, unless otherwise indicated.

\title{
Radiative corrections to the long-wavelength optical-mode spectrum of the electron-phonon model: Absence of mode-splitting effects and hardening of the mode
}

\section{PLEASE CITE THE PUBLISHED VERSION}

\section{PUBLISHER}

(C) American Physical Society

\section{LICENCE}

CC BY-NC-ND 4.0

\section{REPOSITORY RECORD}

Alexandrov, A.S., and J.R. Schrieffer. 2019. "Radiative Corrections to the Long-wavelength Optical-mode Spectrum of the Electron-phonon Model: Absence of Mode-splitting Effects and Hardening of the Mode". figshare. https://hdl.handle.net/2134/1316. 


\title{
Radiative corrections to the long-wavelength optical-mode spectrum of the electron-phonon model: Absence of mode-splitting effects and hardening of the mode
}

\author{
A. S. Alexandrov \\ Department of Physics, Loughborough University, Loughborough LE11 3TU, United Kingdom \\ J. R. Schrieffer \\ National High Magnetic Field Laboratory and Department of Physics, Florida State University, 1800 E. Paul Dirac Drive, \\ Tallahassee, Florida 32306 \\ (Received 21 July 1997)
}

\begin{abstract}
Renormalization of the phonon spectrum of the Einstein form due to a weak coupling with electrons is calculated. No splitting of the phonon mode is found. A $q$-dependent hardening of the long-wave optical phonons contrasts with the softening of acoustic phonons in a weakly coupled electron-phonon system and with the softening of all phonons in the strong-coupling polaronic regime. [S0163-1829(97)02646-5]
\end{abstract}

The problem of coupled electrons and phonons was formulated by Migdal ${ }^{1}$ and by Engelsberg and Schrieffer ${ }^{2}$ for the Debye (acoustic) and Einstein (molecular) phonons, respectively. As Migdal showed, one can omit the vertex corrections for Debye phonons arriving at the renormalized sound velocity $\tilde{s}=s(1-2 \lambda)^{1 / 2}$, where $\lambda$ is the coupling constant. However, in many doped semiconductors, there are optical phonons which are practically dispersionless in the long-wave limit, $\omega(\mathbf{q})=\omega$. In this case the vertex corrections appear to be important when the phonon's phase velocity is of the order or greater than the Fermi velocity, i.e., $\omega$ $\geqslant|\mathbf{q}| v_{F},{ }^{2}$ so that Migdal's theorem does not hold even for weak coupling, $\lambda<1$. Adopting the ladder approximation for the vertex function and expanding it for small $|\mathbf{q}|$ as a power series in $|\mathbf{q}|^{2}$, it was found that the irreducible polarizability $P(q)$ has a pole at $q_{0}=\omega:^{2}$

$$
P(q)=\frac{N}{3}\left(\frac{v_{F}|\mathbf{q}|}{q_{0}}\right)^{2} \frac{2+3 g^{2} N / \omega^{2}-g^{2} N /\left(\omega^{2}-q_{0}^{2}\right)}{\left(1+g^{2} N / \omega^{2}\right)^{2}},
$$

with $N=m p_{F} / 2 \pi^{2}$ the density of states at the Fermi level, $p_{F}=m v_{F}$, and $g$ the electron-phonon coupling constant. Here and below $q \equiv\left(\mathbf{q}, q_{0}\right)$ and $\hbar=c=1$. This expression can be simplified near the pole $q_{0}=\omega$ and $\lambda \equiv g^{2} N / \omega^{2} \ll 1$. Keeping first order terms in $\lambda$, one obtains

$$
P(q) \simeq \frac{2 N}{3}\left(\frac{v_{F}|\mathbf{q}|}{\omega}\right)^{2}\left(1-\frac{\lambda}{4\left(1-q_{0} / \omega\right)}\right),
$$

which yields two poles of the dressed phonon propagator

$$
\widetilde{D}^{-1}(q)=q_{0}^{2}-\omega^{2}-g^{2} P(q)=0 .
$$

The two branch splittings as one increases the momentum transfer to the lattice are

$$
\widetilde{\omega} \simeq \omega \pm \frac{\lambda}{2 \sqrt{3}} v_{F}|\mathbf{q}|
$$

for $|\mathbf{q}| \ll \omega / v_{F}$. Therefore, if an Einstein spectrum is a reasonable choice for the noninteracting phonons, from Eq. (2) one would expect to observe the two branches splitting at finite $|\mathbf{q}|{ }^{2}$ Later on, several authors ${ }^{3-7}$ discussed the vertex corrections in connection with the superconducting $T_{c}$. In particular, Grimaldi et al. ${ }^{7}$ obtained a strong enhancement of $T_{c}$ from the first-order vertex correction for a long-range electron-phonon interaction.

In this paper we recalculate the irreducible polarizability and show that the pole of $P(q)$ in Eq. (1) is an artifact of the expansion in powers of $|\mathbf{q}|$ of the vertex function prior to the integration over the frequency and momentum of the polarization loop. As a result, there is only a hardening of the Einstein phonon mode without any splitting in the weakcoupling regime contrasting with the softening in the strongcoupling polaronic regime, $\lambda>1 .^{8}$ Thus the vertex corrections play no qualitative role in the renormalization of the Einstein phonons.

To illustrate the point, it is sufficient to calculate the zeroth- and first-order diagrams in powers of $\lambda$ as shown in Figs. 1(a) and 1(b), respectively, in a weak-coupling regime, $\lambda \ll 1$. The zeroth-order electronic propagator is determined as

$$
G^{-1}(p)=p_{0}-\xi+i \delta \operatorname{sgn}(\xi) \equiv p_{0}-\xi+i \delta \operatorname{sgn}\left(p_{0}\right),
$$

where $\xi=v_{F}\left(|\mathbf{p}|-p_{F}\right)$ and $p \equiv\left(\mathbf{p}, p_{0}\right)$. The zeroth-order polarizability, Fig. 1(a), is

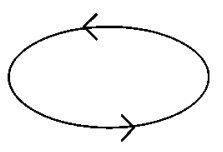

a

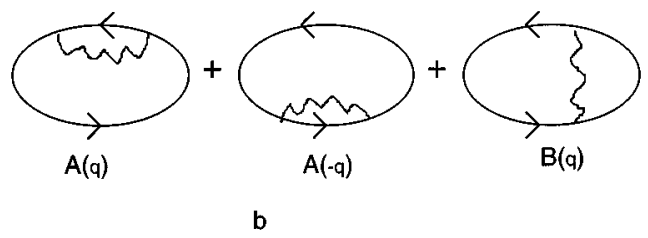

FIG. 1. Zeroth (a) and first order (b) in $\lambda$ irreducible polarizabilities. 


$$
P^{(0)}(q)=-2 i \int \frac{d^{4} p}{(2 \pi)^{4}} G(p+q) G(p)=-i \frac{N}{2 \pi} \int_{-1}^{1} d x \iint \frac{d \xi d p_{0}}{\left[p_{0}+q_{0}-\xi-v_{F}|\mathbf{q}| x+i \delta \operatorname{sgn}\left(p_{0}+q_{0}\right)\right]\left[p_{0}-\xi+i \delta \operatorname{sgn}\left(p_{0}\right)\right]}
$$

with $\delta=+0$. The result depends on the order of integrations if the limits for $\xi$ are extended up to $\pm \infty$. Therefore the frequency $\left(p_{0}\right)$ integral should be calculated first. For $|\mathbf{q}|$ $<q_{0} / v_{F}$, we obtain

$$
P^{(0)}(q)=N\left[\frac{q_{0}}{v_{F}|\mathbf{q}|} \ln \left(\frac{q_{0}+v_{F}|\mathbf{q}|}{q_{0}-v_{F}|\mathbf{q}|}\right)-2\right]
$$

which is the same as Eqs. (1) and (2) with $\lambda=0$ in the long-wave limit, $|\mathbf{q}| \ll q_{0} / v_{F}$. However, the straightforward calculation of the first-order polarizability, Fig. 1(b), leads us to a qualitatively different result compared with Eq. (1) or (2). There are two contributions of the first order from the self-energy effect $(A)$ and one from the vertex correction $(B)$ :

$$
P^{(1)}(q)=A(q)+A(-q)+B(q)
$$

where

$$
A(q)=-2 i \int \frac{d^{4} p}{(2 \pi)^{4}} G^{2}(p) G(p+q) \Sigma(p)
$$

and

$$
\begin{aligned}
B(q)= & 2 g^{2} \iint \frac{d^{4} p d^{4} p^{\prime}}{(2 \pi)^{8}} G(p+q) G(p) G\left(p^{\prime}\right) \\
& \times G\left(p^{\prime}+q\right) D\left(p-p^{\prime}\right),
\end{aligned}
$$

with the bare phonon propagator $D^{-1}(q)=q_{0}^{2}-\omega^{2}+i \delta$. The electron self-energy $\Sigma(p)$ was calculated in Ref. 2 as

$$
\Sigma(p)=-\frac{g^{2} N}{2 \omega}\left(\ln \frac{\left|p_{0}+\omega\right|}{\left|p_{0}-\omega\right|}+i \pi \operatorname{sgn}\left(p_{0}\right) \Theta\left(\left|p_{0}\right|-\omega\right)\right)
$$

where $\Theta(x)=1$ for $x>0$ and zero otherwise. The remaining integrals in Eq. (9) lead to

$$
\begin{aligned}
A(q)= & \frac{g^{2} N^{2}}{q_{0}^{2}-\left(v_{F}|\mathbf{q}|\right)^{2}}\left[\ln \frac{\omega^{2}}{\left|\omega^{2}-q_{0}^{2}\right|}-\frac{q_{0}}{\omega} \ln \frac{\left|\omega+q_{0}\right|}{\left|\omega-q_{0}\right|}\right. \\
& \left.-i \pi \frac{\left|q_{0}\right|-\omega}{\omega} \Theta\left(\left|q_{0}\right|-\omega\right)\right] .
\end{aligned}
$$

By integrating in Eq. (10) first over momenta and then over frequencies, we obtain

$$
\begin{aligned}
B(q)= & -\frac{g^{2} N^{2}}{2\left(v_{F}|\mathbf{q}|\right)^{2}} \ln ^{2}\left(\frac{q_{0}+v_{F}|\mathbf{q}|}{q_{0}-v_{F}|\mathbf{q}|}\right)\left[\ln \frac{\omega^{2}}{\omega^{2}-q_{0}^{2}}\right. \\
& \left.-\frac{q_{0}}{\omega} \ln \frac{\left|\omega+q_{0}\right|}{\left|\omega-q_{0}\right|}-i \pi \frac{\left|q_{0}\right|-\omega}{\omega} \Theta\left(\left|q_{0}\right|-\omega\right)\right] .
\end{aligned}
$$

As a result, the first-order polarizability, Eq. (8), is given by

$$
\begin{aligned}
P^{(1)}(q)= & 2 g^{2} N^{2}\left[\frac{1}{q_{0}^{2}-\left(v_{F}|\mathbf{q}|\right)^{2}}-\left(2 v_{F}|\mathbf{q}|\right)^{-2}\right. \\
& \left.\times \ln ^{2}\left(\frac{q_{0}+v_{F}|\mathbf{q}|}{q_{0}-v_{F}|\mathbf{q}|}\right)\right]\left[\ln \frac{\omega^{2}}{\left|\omega^{2}-q_{0}^{2}\right|}-\frac{q_{0}}{\omega}\right. \\
& \left.\times \ln \frac{\left|\omega+q_{0}\right|}{\left|\omega-q_{0}\right|}-i \pi \frac{\left|q_{0}\right|-\omega}{\omega} \Theta\left(\left|q_{0}\right|-\omega\right)\right] .
\end{aligned}
$$

Near $q_{0}=\omega$ we obtain in the long-wave limit

$$
P(q)=P^{(0)}(q)+P^{(1)}(q) \simeq \frac{2 N}{3}\left(\frac{v_{F}|\mathbf{q}|}{\omega}\right)^{2}(1-2 \lambda \ln 2),
$$

which is drastically different from Eq. (2). There is no pole in the polarizability, and consequently there is no splitting of the Einstein phonon. There are no poles in higher orders either. The absence of any extra branches of collective excitations is a consequence of the conservation of the number of degrees of freedom in a coupled electron-phonon system. Because the Coulomb repulsion is absent from our consideration, there is no other collective excitation except the dressed Einstein mode. The vertex and self-energy corrections are negligible as one can see from Eq. (15) if $\lambda \ll 1$. There is only a hardening of the renormalized optical phonons depending on the momentum as $|\mathbf{q}|^{2}$ :

$$
\widetilde{\omega} \simeq \omega+\frac{\lambda}{3} \frac{\left(v_{F}|\mathbf{q}|\right)^{2}}{\omega} .
$$

The hardening, Eq. (16), contrasts with Migdal's softening of acoustic phonons ${ }^{1}$ and with the softening of all phonons in the strong-coupling polaronic regime. ${ }^{8}$ This contrasting behavior is the result of the familiar repulsion of energy levels under any perturbation. The acoustic phonon energy $s q$ is well below the characteristic energy $v_{F} q$ of the electron-hole continuum, so that if these two excitations are coupled, the lowest acoustic phonon level shifts downwards. On the contrary, the energy of the long-wave optical phonon is well above the electron-hole continuum, $\omega \gg v_{F} q$, and so the upper optical phonon level shifts upwards. In the polaronic 
regime the spectral width of the polaron spectral function is about $g^{2} / \omega^{2}$. It plays the role of an electron-hole continuum being much wider than the width of the phonon spectral function. This is why in the strong-coupling polaronic regime the lowest phonon level is shifted down by the phononpolaron coupling. 8

In conclusion, we have calculated the electronic polarizability of a coupled electron-phonon system including the first-order corrections in powers of $\lambda$. These corrections have no role to play while $\lambda \ll 1$. We have found a $q$-dependent hardening of the Einstein phonons rather than their splitting. The hardening in the weak-coupling regime and the softening in the strong-coupling polaronic regime of optical phonons would allow one to distinguish these two regimes experimentally in doped semiconductors.

J.R.S. acknowledges support of this work by DOE Grant No. DE-FG05-94ER45518.
${ }^{1}$ A. B. Migdal, Zh. Eksp. Teor. Fiz. 34, 1438 (1958).

${ }^{2}$ S. Engelsberg and J. R. Schrieffer, Phys. Rev. 131, 993 (1963).

${ }^{3}$ P. B. Allen and B. Mitrovic, in Solid State Physics, edited by H. Ehrenreich, F. Seitz, and D. Turnbull (Academic, New York, 1982), Vol. 37, p. 1.

${ }^{4}$ High Temperature Superconductivity, edited by V. L. Ginzburg and D. A. Kirzhnits (Consultant Bureau, New York, 1982).
${ }^{5}$ M. Grabowsky and L. J. Sham, Phys. Rev. B 29, 6132 (1984).

${ }^{6}$ Y. Takada, J. Phys. Chem. Solids 54, 1779 (1993).

${ }^{7}$ C. Grimaldi, L. Pietronero, and S. Srassler, Phys. Rev. Lett. 75, 1158 (1995); L. Pietronero, S. Strassler, and C. Grimaldi, Phys. Rev. B 52, 10516 (1995).

${ }^{8}$ A. S. Alexandrov, Phys. Rev. B 46, 2838 (1992); A. S. Alexandrov, H. Capellmann, and U. Gobel, ibid. 46, 4374 (1992). 